\title{
Comunicación y transferencia de conocimiento en los acuerdos de cooperación: una aplicación en la franquicia
}

\author{
Fernández-Monroy, Margarita* \\ Bolívar-Cruz, Alicia M.** \\ Galván-Sánchez, Inmaculada***
}

\section{Resumen}

El propósito de este trabajo es analizar la influencia de la comunicación sobre la transferencia de conocimiento satisfactoria entre los socios de un acuerdo de cooperación. Se trata de un estudio empírico en los sistemas de franquicia que operan en España, centrándose el análisis en la relación franquiciador-franquiciado. Como herramienta metodológica se empleó un cuestionario estructurado autoadministrado dirigido al director general o, en su caso, al responsable de la expansión de la franquicia. Los resultados ponen de manifiesto que una adecuada comunicación influye positivamente en una transferencia de conocimiento satisfactoria. Asimismo, se encontró que la calidad de la relación entre las partes ejerce un papel mediador entre la comunicación y dicha transferencia. Como contribución se señala la necesidad de favorecer adecuados flujos de información y una comunicación de calidad, así como desarrollar una cultura basada en la confianza, el compromiso y el relacionismo entre ambas partes. En cuanto a las implicaciones prácticas, el trabajo proporciona a los gerentes de las franquicias unas pautas para generar una transferencia de conocimiento satisfactoria.

Palabras clave: Comunicación, transferencia de conocimiento, calidad de la relación, franquicia.

Recibido: 31-10-12. Aceptado: 02-02-13

* Doctora por la Universidad de Las Palmas de Gran Canaria. Profesora del Departamento de Economía y Dirección de Empresas. Facultad de Economía, Empresa y Turismo. Universidad de Las Palmas de Gran Canaria. España, e-mail: mfernandez@dede.ulpgc.es. Autora para correspondencia.

** Doctora por la Universidad de Las Palmas de Gran Canaria. Profesora del Departamento de Economía y Dirección de Empresas. Facultad de Economía, Empresa y Turismo. Universidad de Las Palmas de Gran Canaria. España, e-mail: igalvan@dede.ulpgc.es.

*** Doctora por la Universidad de Las Palmas de Gran Canaria. Profesora del Departamento de Economía y Dirección de Empresas. Facultad de Economía, Empresa y Turismo. Universidad de Las Palmas de Gran Canaria. España, e-mail: abolivar@dede.ulpgc.es. 


\title{
Communication and Knowledge Transfer in Cooperation Agreements: An Application to Franchises
}

\begin{abstract}
The purpose of this study is to analyze the influence of communication on satisfactory knowledge transfer among participants in a cooperation agreement. This is an empirical study about franchise systems operating in Spain, centered on analyzing the relation of the franchiser to the franchise operator. A self-administered, structured questionnaire was used as a methodological tool, addressed to the general director or, as applicable, to the person responsible for expanding the franchise. Results reveal that appropriate communication has a positive influence on satisfactory knowledge transfer. Likewise, it was found that the quality of the relationship between the parties exercises a mediating role between communication and the aforementioned transfer. The need to favor appropriate information flow and quality communication is pointed out as a contribution of this research, as is developing a culture based on confidence, commitment and relationism between the two parties. In terms of practical implications, the work offers franchise managers some guidelines for generating satisfactory knowledge transfer.
\end{abstract}

Keywords: Communication, knowledge transfer, relationship quality, franchise.

\section{Introducción}

Desde el enfoque de la gestión del conocimiento, los acuerdos de cooperación constituyen una vía para la creación y transferencia del mismo (Peña y Aranguren, 2002), lo que permite la mejora de la competitividad y la performance de las partes implicadas. En efecto, el desarrollo de acuerdos de cooperación es una respuesta a la necesidad de acceder al conocimiento como recurso estratégico (Benavides, 2011), que de otro modo sería muy difícil de alcanzar.

Uno de los principales objetivos estratégicos de la cooperación es el acceso a las competencias de los socios (Sakakibara, 1997; Mothe y Quélin, 2000; Bayona et al., 2001) y la generación de conocimientos, habilidades y competencias nuevos, además de la propia creación de valor que se genera a partir de los resultados del proceso de cooperación. Las empresas aumentan así el valor de sus activos al adquirir conocimientos tácitos de sus socios (Cassiman, 1999). La cooperación surge, por tanto, como una de las fórmulas más dinámicas para reforzar y completar los recursos limitados de las empresas. Así, las empresas buscan de sus socios aquellos recursos y capacidades de los que carecen, maximizando el valor empresarial a través de la combinación efectiva de los recursos del socio y explotando las complementariedades (Kogut y Singh, 1988; Gulati, 1995; Santamaría y Rialp, 2007). 
En consecuencia, la literatura evidencia la importancia del estudio de la transferencia de conocimiento en los acuerdos de cooperación entre organizaciones, que se formalizan como modo de acceder a dichos recursos y capacidades. Siguiendo a Williams y Gibson (1990) y Albino et al. (2001), la transferencia de conocimiento se concibe como un proceso de comunicación, en el que los gaps entre los entornos del emisor y del receptor afectarán a la eficiencia de la comunicación entre ambos y a la eficacia global de proyecto de transferencia. De esta forma, la transferencia de conocimiento dependerá de la facilidad con la que éste pueda ser transportado, interpretado y asimilado (Hamel et al., 1989).

Ahora bien, la transferencia de conocimiento no implica una completa réplica del mismo ya que, como explican Pedersen et al. (2003), lo que se transfiere no es el conocimiento subyacente sino una aplicación del mismo en forma de una solución a un problema específico. La cuantificación de los resultados derivados del proceso de transferencia de conocimiento resulta complicada debido a que comprende múltiples dimensiones. En los estudios que abordan el éxito de la transferencia de conocimiento se han medido cuestiones como, por ejemplo, el coste y el tiempo necesario para la transferencia, el logro de los objetivos, los cambios que la transferencia generan en la performance del receptor o la satisfacción de los usuarios (Teece, 1977; Reddy y Zhao, 1990; Leonard-Barton y Sinha, 1993; Szulanski, 1995; Zander y Kogut, 1995; Cummings y Teng, 2003), siendo la satisfacción la medida que se va a utilizar en este trabajo.
Al respecto, Mohr y Spekman (1994) señalan que la satisfacción es un importante indicador de los resultados de la cooperación, donde la disponibilidad sistemática de información permite a las partes completar las tareas más eficazmente. En efecto, tal y como señala Cepeda (2003:205), el flujo de información "[...] permite a las empresas [que participan en una relación de intercambio] ser más rápidas y flexibles para adquirir ventajas competitivas, acceder a oportunidades de negocio y evitar equivocaciones potencialmente costosas". Así pues, la comunicación constituye un elemento clave para generar una transferencia de conocimiento satisfactoria.

El principal objetivo de este trabajo es analizar la influencia de la comunicación sobre la transferencia de conocimiento satisfactoria entre los socios de un acuerdo de cooperación. Para ello se presentan, en primer lugar, los fundamentos teóricos más destacados que permitan abordar la relación entre las variables objeto de estudio. Posteriormente, se realiza una aplicación empírica en los sistemas de franquicia, como ejemplo de acuerdos de cooperación, considerando las relaciones que mantiene el franquiciador con sus franquiciados.

La metodología empleada consiste en un estudio empírico en los sistemas de franquicia que operan en España. Al objeto de determinar la población, se decidió contemplar aquellos sistemas de franquicia que la Asociación Española de Franquiciadores (AEF) consideraba como tales. Una vez obtenida la relación de estas franquicias, se procedió a consultar diversas fuentes de información secundarias (guías de franquicias, bases de datos 
electrónicas, páginas web propias de las enseñas) con el fin de disponer de la información necesaria para contactar con cada una de ellas. Finalmente la población quedó constituida por un total de 592 franquicias.

Se diseñó un cuestionario que fue sometido a un pretest entre expertos académicos y profesionales del campo de la franquicia, así como con diez franquiciadores de la población objeto de estudio, con enseñas incluidas en diversos sectores de actividad. El cuestionario se envió por correo postal a toda la población para ser contestado por el director general o, en su caso, el responsable de la expansión de la franquicia para abordar el análisis de la influencia de la comunicación sobre la transferencia de conocimiento satisfactoria.

Tras un proceso de seguimiento continuo, que supuso varias oleadas de envíos de cuestionarios por fax y correo electrónico, se obtuvo un total de 98 cuestionarios válidos, lo que representa una tasa de respuesta del $16,55 \%$. Con la información recabada se contrastó el modelo propuesto de transferencia de conocimiento en los sistemas de franquicia, a través de análisis factoriales y ecuaciones estructurales.

\section{La transferencia de conocimiento en los acuerdos de cooperación}

La naturaleza de la cooperación entre las partes puede ser una barrera para la transferencia efectiva de conocimiento (Goh, 2002). Así pues, si la relación entre ambas es distante o la comunicación es difícil, es menos probable que se produzca la transferencia de forma satisfactoria. Al respecto, la investigación empírica ha evidenciado la importancia de la comunicación como factor crítico en los resultados organizativos, siendo un elemento esencial en un contexto de cooperación para alcanzar los beneficios esperados. En efecto, "[...] la relación comercial más cuidadosamente diseñada se derrumbará sin una buena y frecuente comunicación entre las organizaciones involucradas" (Cepeda, 2003:204).

De este modo, tal y como señalan De Wulf et al. (2001), la comunicación aumenta la probabilidad de descubrir comportamientos que generan recompensas, mejora la predicción de los comportamientos de la otra parte y clarifica los roles de cada una, conduce al descubrimiento de similitudes, y anima sentimientos de confianza y cercanía. En este sentido, es necesario distinguir dos dimensiones de la comunicación: por una parte, el intercambio de información y, por otra, la calidad con la que se desarrolla el proceso de comunicación. En términos generales, aspectos como la frecuencia de los contactos, la bidireccionalidad de los flujos de comunicación y las estrategias de influencia basadas en el intercambio de información generan una comunicación de colaboración (Mohr et al., 1996).

Con respecto a la segunda dimensión, Mohr y Spekman (1994) destacan el papel clave que desempeña la calidad de la comunicación en la transmisión de información, definida como el grado en que los integrantes de la cooperación perciben que los flujos de comunicación son adecuados, oportunos (se producen a tiempo), precisos, completos y creíbles (Daft y Lengel, 1986). 
En la literatura se muestra que niveles elevados de estas dimensiones de la comunicación se asocian con alianzas más exitosas, en términos de desempeño y de satisfacción con diversos aspectos de la relación (Mohr y Nevin, 1990; Mohr y Spekman, 1994; Boyle y Dwyer, 1995; Mohr et al., 1996; Simpson y Mayo, 1997; Selnes, 1998), entre otras. Así, en las relaciones de negocio en las que existe una gran interdependencia entre los socios, es fundamental un elevado nivel de comunicación para lograr una mejor coordinación y propiciar la transferencia de conocimiento (Dawson, 2000; Cabrera y Cabrera, 2002; Rodríguez Orejuela, 2007).

Fruto de esta argumentación, se establece la influencia directa y positiva de la comunicación sobre la transferencia de conocimiento, que se concreta en dos hipótesis que reflejan el efecto directo entre cada una de las dimensiones de la comunicación (el flujo de información y la calidad de la comunicación de los socios) y la variable dependiente:

H1 El flujo de información tiene una influencia directa y positiva sobre una satisfactoria transferencia de conocimiento.

H2 La calidad de la comunicación tiene una influencia directa y positiva sobre una satisfactoria transferencia de conocimiento.

Por otra parte, el desarrollo de una atmósfera de confianza, compromiso organizativo y apoyo mutuo puede mejorar el flujo de conocimiento entre los socios, lo que pone de manifiesto el importante papel que desempeñan estas variables en la transferencia de conocimiento (Cabrera y Cabrera, 2002; Feng y Zhang, 2007). Es precisamente una adecuada comunica- ción el elemento que contribuye a crear una atmósfera de apoyo y respeto mutuos, a la vez que pone de relieve los intereses compartidos y los objetivos comunes (Mohr et al., 1996). En consecuencia, la comunicación es un importante factor en el desarrollo de las relaciones y las evaluaciones de la calidad de la relación (Mohr y Sohi, 1995). En esta misma línea, Jap et al. (1999) reconocen que la comunicación exhibida durante la interacción entre ambas partes también afecta significativamente a la calidad de la relación.

De ahí surge el importante papel de la calidad de la relación como variable que media entre la comunicación y la transferencia de conocimiento satisfactoria. Los investigadores han explicado la calidad de la relación en términos muy diversos (Jap et al., 1999; Hausman, 2001), aunque en el presente estudio, dado el énfasis existente en la literatura al respecto, se considerará que la calidad de la relación viene determinada por la confianza entre las partes de la colaboración, el nivel de compromiso mutuo y el grado de relacionismo entre los socios.

La confianza se ha descrito fundamentalmente a través de dos componentes diferenciados: la credibilidad y la benevolencia (Ganesan, 1994; Mayer et al., 1995; Doney y Cannon, 1997; Das y Teng, 1998; Siguaw et al, 1998; Baker et al., 1999; Gilliland y Bello, 2002). El primer componente hace referencia al grado en que una de las partes cree que la otra tiene la formación necesaria para desempeñar el trabajo eficaz y responsablemente (Ganesan, 1994), mientras que el segundo, la benevolencia, se entiende como el grado en que un socio de la relación está real- 
mente interesado en el bienestar del otro y está motivado a buscar el beneficio conjunto (Doney y Cannon, 1997).

En cuanto al compromiso, en la literatura se distinguen dos componentes: el afectivo y el calculado (Brown et al., 1995; Geyskens et al., 1996; Gilliland y Bello, 2002; Verhoef et al., 2002). El compromiso afectivo se refiere al deseo de continuar una relación a causa de un sentimiento de afecto hacia el socio (Kumar et al, 1995) y el compromiso calculado hace referencia al grado en que los miembros de la relación perciben la necesidad de mantenerla debido a los elevados costes de cambio o de terminación anticipada asociados al abandono (Geyskens et al., 1996).

Respecto al relacionismo, se identifican dos de las dimensiones más presentes en las investigaciones (Boyle et al., 1992; Heide y John, 1992; Simpson y Mayo, 1997; Siguaw et al., 1998; Paswan y Young, 1999; Cannon et al., 2000; Jap y Ganesan, 2000; Hausman, 2001; Bello et al, 2003): la solidaridad y la mutualidad. La solidaridad hace referencia al grado en que las partes consideran que la relación de intercambio es importante en sí y por sí misma, dado que creen que el éxito procede de trabajar juntas de forma cooperativa en vez de competir una contra otra; mientras que la mutualidad recoge la actitud de que el éxito de cada parte está en función del éxito del otro, expresando un sentimiento de responsabilidad conjunta (Cannon et al., 2000).

En la literatura se muestra que niveles elevados de las dimensiones de la comunicación se asocian con la calidad de la relación, en términos de confianza (Morgan y Hunt, 1994; Doney y Cannon, 1997; Simpson y Mayo, 1997; Selnes,
1998), compromiso (Anderson y Weitz, 1992; Mohr et al, 1996; Simpson y Mayo, 1997) y normas relacionales (Boyle et al., 1992), entre otras. Asimismo, se evidencia que la confianza y el compromiso son variables esenciales para una cooperación satisfactoria (Mohr y Spekman, 1994; Siguaw et al., 1998; Sarkar et al., 2001; Montoro-Sánchez et al., 2010) y que las normas relacionales tienen un efecto positivo sobre el resultado (Lusch y Brown, 1996; Siguaw et al., 1998; Cannon y Perreault, 1999; Cannon et al., 2000; Hausman, 2001; Bello et al., 2003).

Así pues, a partir de esta argumentación, se observa la influencia indirecta y positiva que ejerce la comunicación, mediada por la calidad de la relación, sobre una satisfactoria transferencia de conocimiento, y que se concreta en las siguientes dos hipótesis:

H3 El flujo de información tiene una influencia indirecta y positiva, mediada por la calidad de la relación, sobre una satisfactoria transferencia de conocimiento. Esto es, el flujo de información tiene un efecto directo sobre la calidad de la relación, la cual, a su vez, afecta a una satisfactoria transferencia de conocimiento. H4 La calidad de la comunicación tiene una influencia indirecta y positiva, mediada por la calidad de la relación, sobre una satisfactoria transferencia de conocimiento. Esto es, la calidad de la comunicación tiene un efecto directo sobre la calidad de la relación, la cual, a su vez, afecta a una satisfactoria transferencia de conocimiento.

En definitiva, a través de las hipótesis planteadas, se trata de analizar la influencia de la comunicación sobre la transferencia de conocimiento satisfactoria entre los so- 
cios de un acuerdo de cooperación. De manera más específica, se pretende examinar el efecto que ejerce la comunicación (que incluye el flujo de información y la calidad de la comunicación), tanto directa como indirectamente mediante la calidad de la relación, sobre una satisfactoria transferencia de conocimiento, tal y como se refleja en el Diagrama 1.

\section{La comunicación como determinante de la trasferencia de conocimiento satisfactoria: evidencia empírica}

Como ya se ha señalado, el contexto de aplicación de este trabajo se sitúa en los sistemas de franquicia. Su elección como objeto de estudio se ha visto motivada, entre otros aspectos, por el hecho de que en los últimos años el sistema de la franquicia ha adquirido una gran importancia como modelo de negocio, con ventajas tanto para el franquiciador como para el franquiciado (Bordonaba et al.,
2009), siendo trascendentales sus implicaciones económicas en el mercado.

La franquicia es un sistema de cooperación entre empresas diferentes que supone la expansión de una idea o concepto de éxito (Díez y Galán, 1998). En este sentido, la escasez de recursos ha sido uno de los argumentos esgrimidos en la literatura a la hora de explicar los motivos por los que las empresas eligen crecer a través del sistema de franquicia en vez de utilizar establecimientos propios. De este modo, diversos autores (Oxenfeldt y Kelly, 1969; Norton, 1988; Combs y Castrogiovanni, 1994) exponen dicho argumento basándose en la idea de que los franquiciadores utilizan el sistema de franquicia para lograr el acceso a determinados recursos necesarios para acelerar el crecimiento de la empresa (capital financiero, capital humano, talento directivo, conocimiento de mercado).

Al basarse el crecimiento del negocio en empresas independientes, es fundamental desarrollar la cooperación entre estos socios sobre unas bases sóli-

\section{Diagrama 1 \\ Modelo propuesto}

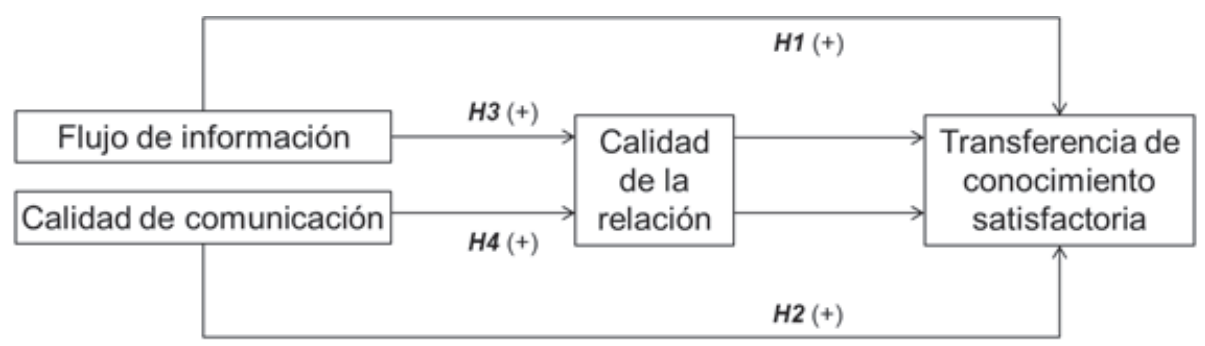

Fuente: Elaboración propia. 
das, asentadas en la mutua confianza y en un reparto equitativo de los beneficios que se obtienen de la franquicia (Díez y Galán, 1998). Por tanto, además de la relación contractual entre franquiciador y franquiciado, es preciso destacar la relación de cooperación entre ambos, que puede determinar el éxito del negocio. Ello implica el desarrollo de vínculos que facilitan la creación, el mantenimiento y el incremento del valor en la relación franquiciador-franquiciado, entre los que cabe citar la comunicación, la confianza y el compromiso (Bermúdez, 2002; Bordonaba y Polo, 2004). Así, en el marco de esta cooperación se facilita el proceso de transferencia de conocimiento, por ejemplo cuando el franquiciador transmite al franquiciado los objetivos, las instrucciones y toda la información precisa para la adecuada gestión del negocio (Rodríguez et al., 2008).

Es en el contexto de la relación entre el franquiciador y sus franquiciados en el que se lleva a cabo el contraste del modelo propuesto. Las medidas de los constructos de dicho modelo se diseñaron a partir de la revisión de la literatura y de las entrevistas exploratorias realizadas a expertos tanto académicos como profesionales del campo. Fruto de estas actividades, se definieron los instrumentos de medida relacionados con la comunicación -que incluye el flujo de información y la calidad de comunicación-, la calidad de la relación entre los socios (franquiciador y franquiciados), así como la transferencia de conocimiento satisfactoria. En el Cuadro 1 , se recogen los ítems definitivos integrantes de cada uno de los constructos incluidos en el cuestionario, valorados en escalas tipo Likert de 7 puntos.
Antes de evaluar el modelo estructural propuesto, se procedió a analizar la validez y fiabilidad de las escalas de medida utilizadas. Con tal objeto, se aplicó, en primer lugar, análisis factoriales exploratorios para depurar y conocer la naturaleza dimensional de las escalas; en segundo lugar, análisis factoriales confirmatorios con el propósito de confirmar los resultados obtenidos, utilizando para ello ecuaciones lineales estructurales, $y$, finalmente, el coeficiente de fiabilidad compuesta y el análisis de la varianza extraída para evaluar la fiabilidad de las escalas empleadas, cuyos niveles superaban en todos los casos los valores recomendados. El análisis de los datos se llevó a cabo con los paquetes estadísticos SPSS y AMOS.

En la Tabla 1, se recogen los resultados de los análisis factoriales confirmatorios de los constructos relativos a la comunicación: flujo de información y calidad de la comunicación. Para evaluar el ajuste del modelo de medida de los constructos se emplearon los siguientes índices: (1) el ratio de verosimilitud Chi-cuadrado (CMIN), el índice de bondad del ajuste (GFI) y el error cuadrático medio de aproximación (RMSEA) como medidas absolutas de ajuste; (2) el índice de bondad del ajuste corregido (AGFI) y el índice del ajuste normal (NFI) como medidas de ajuste incremental, y (3) el índice de ajuste normado de parsimonia (PNFI), el índice de calidad de ajuste de parsimonia (PGFI) y el ratio de Chi-cuadrado sobre los grados de libertad (CMIN/GL) como medidas de ajuste de parsimonia.

Considerando los valores umbrales comúnmente aceptados en la literatura (Hair et al., 1999), se comprueba que 


\section{Cuadro 1 \\ Escalas de medida de los constructos}

\begin{tabular}{|c|c|c|c|}
\hline \multicolumn{2}{|c|}{ VARIABLES } & \multicolumn{2}{|r|}{ ÍTEMS DEFINITIVOS } \\
\hline \multirow{4}{*}{\multicolumn{2}{|c|}{$\begin{array}{l}\text { FLUJO DE } \\
\text { INFORMACIÓN }\end{array}$}} & FLU1 & $\begin{array}{l}\text { Ambos aportamos información estratégica y/u operativa, relacionada con el } \\
\text { negocio de cada uno, cuando beneficia a la relación. }\end{array}$ \\
\hline & & FLU2 & $\begin{array}{l}\text { Nos mantenemos mutuamente informados sobre los acontecimientos o } \\
\text { cambios que puedan afectar a cada una de las partes. }\end{array}$ \\
\hline & & FLU3 & Ambos intercambiamos información frecuentemente. \\
\hline & & FLU4 & $\begin{array}{l}\text { Ambos intercambiamos información utilizando los canales de comunicación } \\
\text { establecidos para ello. }\end{array}$ \\
\hline \multirow{3}{*}{\multicolumn{2}{|c|}{$\begin{array}{l}\text { CALIDAD DE LA } \\
\text { COMUNICACIÓN }\end{array}$}} & CA1 & Inadecuada/Adecuada \\
\hline & & CA2 & Incompleta/Completa \\
\hline & & CA3 & No a tiempo/A tiempo \\
\hline \multirow{8}{*}{\multicolumn{2}{|c|}{ Confianza }} & CF1 & Ambos somos honestos y sinceros. \\
\hline & & CF2 & Ambos consideramos que la información que nos intercambiamos es fiable. \\
\hline & & CF3 & $\begin{array}{l}\text { Ambos comunicamos con sinceridad cualquier problema que pueda afectar } \\
\text { a la otra parte. }\end{array}$ \\
\hline & & CF4 & $\begin{array}{l}\text { Ambos consideramos los intereses de la otra parte en la toma de } \\
\text { decisiones importantes. }\end{array}$ \\
\hline & & CF5 & En tiempos difíciles, ambos continuamos confiando el uno en el otro. \\
\hline & & CF6 & Ambos sentimos que cada uno está de parte del otro. \\
\hline & & CF7 & $\begin{array}{l}\text { Ambos estamos dispuestos a ofrecer asistencia y ayuda cuando las } \\
\text { circunstancias lo requieren. }\end{array}$ \\
\hline & & CF8 & En términos generales, ambos confiamos el uno en el otro. \\
\hline$\sum_{\vec{w}}$ & \multirow{5}{*}{ Compromiso } & CP1 & Ambos tenemos un fuerte sentido de lealtad hacia el otro. \\
\hline$\frac{\vec{r}}{\square}$ & & $\mathrm{CP} 2$ & $\begin{array}{l}\text { Ninguno está interesado en ofertas de otras empresas que puedan } \\
\text { reemplazar a la otra parte. }\end{array}$ \\
\hline 㟔 & & $\mathrm{CP} 3$ & Ambos consideramos esta relación como una colaboración a largo plazo. \\
\hline 是 & & $\mathrm{CP} 4$ & Ambos esperamos que esta relación continúe durante mucho tiempo. \\
\hline 曹 & & CP5 & En términos generales, ambos estamos mutuamente comprometidos. \\
\hline 汖 & \multirow{6}{*}{ Relacionismo } & RE1 & Ninguna parte haria nada que perjudicara a la otra. \\
\hline & & RE2 & $\begin{array}{l}\text { Los problemas que surgen se tratan como responsabilidades conjuntas } \\
\text { más que individuales. }\end{array}$ \\
\hline & & RE3 & $\begin{array}{l}\text { Ambos estamos dispuestos a efectuar mejoras que puedan beneficiar } \\
\text { nuestra relación como un todo, y no sólo a una de las partes. }\end{array}$ \\
\hline & & RE4 & Cada parte se preocupa por la rentabilidad de la otra. \\
\hline & & RE5 & Ambas partes se ayudan mutuamente. \\
\hline & & RE6 & $\begin{array}{l}\text { Ambos vemos esta relación como un "esfuerzo de cooperación" más que } \\
\text { como un "conjunto de transacciones individuales". }\end{array}$ \\
\hline \multirow{4}{*}{\multicolumn{2}{|c|}{$\begin{array}{l}\text { TRANSFERENCIA } \\
\text { DE } \\
\text { CONOCIMIENTO } \\
\text { SATISFACTORIA }\end{array}$}} & TR1 & $\begin{array}{l}\text { Si tuviéramos que crear de nuevo una relación de estas caracteristicas, } \\
\text { volveríamos a hacer negocios con las mismas empresas participantes. }\end{array}$ \\
\hline & & TR2 & $\begin{array}{l}\text { En general, desempeñamos bien nuestras respectivas tareas en esta } \\
\text { relación mutua. }\end{array}$ \\
\hline & & TR3 & Esta relación puede ser descrita como de éxito. \\
\hline & & TR4 & $\begin{array}{l}\text { En términos generales, estamos muy satisfechos con la relación que } \\
\text { mantenemos. }\end{array}$ \\
\hline
\end{tabular}

Fuente: Elaboración propia. 
Tabla 1

Modelo de medida del flujo de información y la calidad de la comunicación

\begin{tabular}{lccc}
\hline \multicolumn{1}{c}{ Variables } & $\begin{array}{c}\text { Estimadores } \\
\text { estandarizados }\end{array}$ & Razones críticas & $\begin{array}{c}\text { Significación } \\
(\mathbf{p})\end{array}$ \\
\hline FLU1 $\leftarrow$ Flujo de comunicación & 0,685 & & \\
FLU2 $\leftarrow$ Flujo de comunicación & 0,771 & 6,832 & 0,000 \\
FLU3 $\leftarrow$ Flujo de comunicación & 0,830 & 7,274 & 0,000 \\
FLU4 $\leftarrow$ Flujo de comunicación & 0,886 & 7,623 & 0,000 \\
CA1 $\leftarrow$ Calidad de comunicación & 0,877 & 9,519 & 0,000 \\
CA2 $\leftarrow$ Calidad de comunicación & 0,856 & 9,306 & 0,000 \\
CA3 $\leftarrow$ Calidad de comunicación & 0,806 & & \\
\hline
\end{tabular}

\section{Resultados del ajuste del modelo de medida}

$\mathrm{CMIN}=12,802(p=0,463) \mathrm{GFI}=0,965$ RMSEA $=0,000$

$\mathrm{NFI}=0,969 \mathrm{AGFI}=0,924 \mathrm{PGFI}=0,448 \mathrm{CMIN} / \mathrm{DF}=0,985 \mathrm{PNFI}=0,600$

Fuente: Elaboración propia.

los indicadores de bondad del ajuste son aceptables, dado que las medidas se sitúan alrededor de los límites recomendados, y que todos los pesos de regresión estandarizados presentan razones críticas superiores al valor recomendado de \pm 1,96 . Los resultados de estos análisis ponen de manifiesto que estos constructos son de naturaleza unidimensional, si bien se ha tenido que realizar la confirmación de los modelos de medida de forma simultánea, dado el bajo número de ítems de la calidad de comunicación, que ha imposibilitado su análisis como modelo de medida de forma independiente.

Respecto al constructo calidad de la relación, los resultados de los análisis exploratorios realizados arrojaron la existencia de dos factores en la dimensión confianza (credibilidad y benevolencia), dos factores en la dimensión compromiso (compromiso calculado y compromiso afectivo) y dos factores en la dimensión relacionismo (solidaridad y mutualidad). Seguidamente, se realizó un análisis factorial confirmatorio de segundo orden para cada una de estas dimensiones, obteniéndose resultados satisfactorios y confirmándose la naturaleza bidimensional de las tres dimensiones de la calidad de la relación. Tomando como base los resultados de dichos análisis, se crearon seis indicadores a partir de los estimadores estandarizados, que se utilizaron en el modelo global de medida de la calidad de la relación, cuyos resultados se muestran en la Tabla 2. Estos resultados ponen de manifiesto una bondad del ajuste aceptable y muestran la naturaleza tridimensional del constructo calidad de la relación.

En cuanto al constructo transferencia de conocimiento satisfactoria, el análisis factorial confirmatorio confirma el carácter unidimensional ya obtenido en el análisis factorial exploratorio, tal y como se extrae de los resultados recogidos en 
Tabla 2

Modelo de medida de la calidad de la relación

\begin{tabular}{|c|c|c|c|c|}
\hline \multicolumn{2}{|r|}{ Variables } & \multirow{2}{*}{$\begin{array}{c}\begin{array}{c}\text { Estimadores } \\
\text { estandarizados }\end{array} \\
0,998\end{array}$} & \multirow[t]{2}{*}{$\begin{array}{c}\text { Razones } \\
\text { críticas }\end{array}$} & \multirow[t]{2}{*}{$\begin{array}{c}\text { Significación } \\
\text { (p) }\end{array}$} \\
\hline Confianza & $\leftarrow$ Calidad de la relación & & & \\
\hline Compromiso & $\leftarrow$ Calidad de la relación & 0,971 & 11,398 & 0,000 \\
\hline Relacionismc & $0 \leftarrow$ Calidad de la relación & 0,971 & 12,632 & 0,000 \\
\hline Credibilidad & $\leftarrow$ Confianza & 0,882 & & \\
\hline Benevolencia & $a \leftarrow$ Confianza & 0,916 & 13,876 & 0,000 \\
\hline CF8 & $\leftarrow$ Confianza & 0,935 & 14,597 & 0,000 \\
\hline Comp. Afect. & $\leftarrow$ Compromiso & 0,871 & & \\
\hline Comp. Calcu & l. $\leftarrow$ Compromiso & 0,828 & 10,721 & 0,000 \\
\hline CP5 & $\leftarrow$ Compromiso & 0,840 & 11,010 & 0,000 \\
\hline Solidaridad & $\leftarrow$ Relacionismo & 0,918 & & \\
\hline Mutualidad & $\leftarrow$ Relacionismo & 0,905 & 14,701 & 0,000 \\
\hline RE6 & $\leftarrow$ Relacionismo & 0,763 & 10,021 & 0,000 \\
\hline
\end{tabular}

Resultados del ajuste del modelo de medida

CMIN $=34,303(p=0,079)$ GFI $=0,929$ RMSEA $=0,067$

$\mathrm{NFI}=0,964 \mathrm{AGFI}=0,867 \mathrm{PGFI}=0,496 \mathrm{CMIN} / \mathrm{DF}=1,429 \mathrm{PNFI}=0,643$

Fuente: Elaboración propia.

la Tabla 3, en la que se detalla la especificación del modelo de medida propuesto para este constructo.
Finalmente, en la Tabla 4 se muestran los resultados de los análisis de la fiabilidad compuesta y de la varianza extraí-

Tabla 3

Modelo de medida de la transferencia de conocimiento satisfactoria

\begin{tabular}{lccc}
\hline Variables & $\begin{array}{c}\text { Estimadores } \\
\text { estandarizados }\end{array}$ & $\begin{array}{c}\text { Razones } \\
\text { críticas }\end{array}$ & $\begin{array}{c}\text { Significación } \\
\mathbf{( p )}\end{array}$ \\
\hline TR1 $\leftarrow$ Transferencia de conocimiento satisfactoria & 0,749 & 10,303 & 0,000 \\
TR2 $\leftarrow$ Transferencia de conocimiento satisfactoria & 0,825 & 12,773 & 0,000 \\
TR3 $\leftarrow$ Transferencia de conocimiento satisfactoria & 0,882 & 15,307 & 0,000 \\
TR4 $\leftarrow$ Transferencia de conocimiento satisfactoria & 0,980 & & \\
\hline
\end{tabular}

\section{Resultados del ajuste del modelo de medida}

CMIN $=0,545(p=0,762) \mathrm{GFI}=0,997$ RMSEA $=0,000$

$\mathrm{NFI}=0,998 \mathrm{AGFI}=0,986 \mathrm{PGFI}=0,199 \mathrm{CMIN} / \mathrm{DF}=0,272 \mathrm{PNFI}=0,333$

Fuente: Elaboración propia. 
Tabla 4

Fiabilidad compuesta y varianza extraída

\begin{tabular}{lcc}
\hline \multicolumn{1}{c}{ Variables } & Fiabilidad compuesta & Varianza extraída \\
\hline Flujo de información & 0,873 & 0,634 \\
Calidad de la comunicación & 0,884 & 0,717 \\
Calidad de la relación & 0,981 & 0,814 \\
Transferencia de conocimiento satisfactoria & 0,920 & 0,745 \\
\hline
\end{tabular}

Fuente: Elaboración propia.

da para cada uno de los constructos desarrollados. Tal y como se ha señalado, los niveles obtenidos superaban en todos los casos los valores recomendados, esto es, índices de fiabilidad compuesta superiores a 0,7 y de varianza extraída superiores a 0,5 (Hair et al., 1999).

Por otra parte, al objeto de analizar los factores que inciden en la transferencia de conocimiento satisfactoria, particularmente en lo que respecta a la influencia que ejerce el flujo de información y la calidad de la comunicación tanto directa como indirectamente mediante la calidad de la relación, se ha procedido a estimar el modelo que se ha propuesto utilizando para ello un modelo de ecuaciones estructurales, con el que se dará respuesta al contraste de las hipótesis formuladas.

En la Tabla 5, se recogen los resultados de este modelo estructural, los cuales ponen de manifiesto una bondad del ajuste satisfactoria y que la mayoría de los pesos de regresión estandarizados presentan razones críticas superiores al valor recomendado. Al mismo tiempo, los niveles de las correlaciones múltiples al cuadrado obtenidos reflejan claramente que se trata de un modelo con el que se explica en una proporción considerable la transferencia de conocimiento satisfacto- ria $(84,7 \%)$, así como la calidad de la relación que mantiene el franquiciador con sus franquiciados (62,5\%). Estos resultados evidencian de forma genérica que la comunicación facilita una satisfactoria transferencia de conocimiento entre franquiciador y franquiciado, donde la calidad de la relación existente entre ellos desempeña un importante papel mediador.

El examen pormenorizado de los estimadores estandarizados y de las razones críticas del modelo especificado revela que, en cierta medida, la comunicación entre el franquiciador y sus franquiciados afecta directa y positivamente a la transferencia de conocimiento satisfactoria. Concretamente, se puede aceptar la influencia directa que ejerce el flujo de información sobre dicha transferencia a un $6 \%$ de nivel de significación $(0,205$, $p=0,060$ ), lo que apoya la hipótesis H1. No sucede lo mismo con el efecto directo de la calidad de la comunicación sobre la transferencia de conocimiento, pues no se ha obtenido una relación estadísticamente significativa entre dichas variables $(-0,101, p=0,243)$, lo que supone rechazar la hipótesis $\mathrm{H} 2$.

Asimismo, se contrasta la influencia indirecta de la comunicación sobre la transferencia de conocimiento satisfacto- 


\section{Tabla 5}

Modelo estructural de la influencia de la comunicación sobre la satisfactoria transferencia de conocimiento

\begin{tabular}{|c|c|c|c|}
\hline Variables & $\begin{array}{l}\text { Estimadores } \\
\text { estandarizados }\end{array}$ & $\begin{array}{l}\text { Razones } \\
\text { críticas }\end{array}$ & $\begin{array}{c}\text { Significación } \\
\text { (p) }\end{array}$ \\
\hline Calidad de la relación $\leftarrow$ Flujo de información & 0,621 & 4,504 & 0,000 \\
\hline Calidad de la relación $\leftarrow$ Calidad de comunicación & 0,216 & 1,791 & 0,073 \\
\hline $\begin{array}{l}\text { Transf. conocimiento satisfactoria } \leftarrow \text { Calidad } \\
\text { de relación }\end{array}$ & 0,821 & 8,393 & 0,000 \\
\hline $\begin{array}{l}\text { Transf. conocimiento satisfactoria } \leftarrow \text { Calidad } \\
\text { comunicación }\end{array}$ & $-0,101$ & $-1,168$ & 0,243 \\
\hline $\begin{array}{l}\text { Transf. conocimiento satisfactoria } \leftarrow \text { Flujo } \\
\text { de información }\end{array}$ & 0,205 & 1,880 & 0,060 \\
\hline FLU1 $\leftarrow$ Flujo de información & 0,705 & & \\
\hline FLU2 $\leftarrow$ Flujo de información & 0,788 & 7,251 & 0,000 \\
\hline FLU3 $\leftarrow$ Flujo de información & 0,825 & 7,566 & 0,000 \\
\hline FLU4 $\leftarrow$ Flujo de información & 0,866 & 7,892 & 0,000 \\
\hline CA1 $\leftarrow$ Calidad de comunicación & 0,888 & 9,671 & 0,000 \\
\hline $\mathrm{CA} 2 \leftarrow$ Calidad de comunicación & 0,844 & 9,198 & 0,000 \\
\hline CA3 $\leftarrow$ Calidad de comunicación & 0,804 & & \\
\hline Relacionismo $\leftarrow$ Calidad de la relación & 0,922 & & \\
\hline Compromiso $\leftarrow$ Calidad de la relación & 0,919 & 16,030 & 0,000 \\
\hline Confianza $\leftarrow$ Calidad de la relación & 0,969 & 19,184 & 0,000 \\
\hline TR1 $\leftarrow$ Transferencia de conocimiento satisfactoria & 0,763 & 10,643 & 0,000 \\
\hline TR2 $\leftarrow$ Transferencia de conocimiento satisfactoria & 0,849 & 13,725 & 0,000 \\
\hline TR3 $\leftarrow$ Transferencia de conocimiento satisfactoria & 0,884 & 15,516 & 0,000 \\
\hline TR4 $\leftarrow$ Transferencia de conocimiento satisfactoria & 0,960 & & \\
\hline
\end{tabular}

\section{Resultados del ajuste del modelo de medida}

CMIN $=87,820(p=0,086)$ GFI $=0,890$ RMSEA $=0,049$

$\mathrm{NFI}=0,935 \mathrm{AGFI}=0,837 \mathrm{PGFI}=0,602 \mathrm{CMIN} / \mathrm{DF}=1,237 \mathrm{PNFI}=0,729$

Fuente: Elaboración propia. 
ria, mediada por la calidad de la relación. En otras palabras, la calidad de la relación ejerce un papel mediador en la relación entre cada uno de los constructos que describen la comunicación y la satisfactoria transferencia de conocimiento. Así pues, por una parte, se observa que el flujo de información ejerce un efecto directo sobre la calidad de la relación $(0,621, p=0,000)$, la cual tiene una influencia directa sobre dicha transferencia de conocimiento $(0,821, p=0,000)$, lo que permite aceptar la hipótesis H3. Por otra parte, los resultados muestran que la calidad de la comunicación tiene un efecto directo sobre la calidad de la relación a un $8 \%$ de nivel de significación $(0,216$, $p=0,073$ ), la cual, como ya se ha señalado, afecta directamente a la transferencia de conocimiento. Estos resultados apoyan la hipótesis $\mathrm{H} 4$.
En resumen, tal y como se observa en el Cuadro 2, se confirman todas las hipótesis planteadas, salvo una, la relativa a la influencia directa que la calidad de la comunicación ejerce sobre la transferencia de conocimiento (H2). Ahora bien, no implica que dichas variables no estén relacionadas, sino que la relación no es directa. En efecto, tal y como se confirma con la aceptación de la hipótesis H4, la calidad de la comunicación influye sobre la transferencia de conocimiento de forma indirecta, a través de la calidad de la relación.

A raíz de los resultados obtenidos, se evidencia que el logro de una transferencia de conocimiento satisfactoria depende directamente del flujo de información en la relación franquiciador-franquiciado, es decir, del grado en que franquiciador y franquiciados se intercambian información estratégica

\section{Cuadro 2}

Resumen del contraste de hipótesis

\begin{tabular}{||c|l|c||}
\hline \hline \multicolumn{2}{|c|}{ Hipótesis } & Resultado \\
\hline H1 & $\begin{array}{l}\text { El flujo de información tiene una influencia directa y positiva sobre una } \\
\text { satisfactoria transferencia de conocimiento }\end{array}$ & Confirmada \\
\hline H2 & $\begin{array}{l}\text { La calidad de la comunicación tiene una influencia directa y positiva } \\
\text { sobre una satisfactoria transferencia de conocimiento. }\end{array}$ & Rechazada \\
\hline H3 & $\begin{array}{l}\text { El flujo de información tiene una influencia indirecta y positiva, mediada } \\
\text { por la calidad de la relación, sobre una satisfactoria transferencia de } \\
\text { conocimiento. Esto es, el flujo de información tiene un efecto directo } \\
\text { sobre la calidad de la relación, la cual, a su vez, afecta a una } \\
\text { satisfactoria transferencia de conocimiento. }\end{array}$ & Confirmada \\
\hline H4 & $\begin{array}{l}\text { La calidad de la comunicación tiene una influencia indirecta y positiva, } \\
\text { mediada por la calidad de la relación, sobre una satisfactoria } \\
\text { transferencia de conocimiento. Esto es, la calidad de la comunicación } \\
\text { tiene un efecto directo sobre la calidad de la relación, la cual, a su vez, } \\
\text { afecta a una satisfactoria transferencia de conocimiento. }\end{array}$ & Confirmada \\
\hline
\end{tabular}

Fuente: Elaboración propia. 
y operativa relacionada con el negocio, se mantienen mutuamente informados sobre acontecimientos que puedan ser importantes para el otro socio y, en definitiva, comparten información frecuentemente a través de los canales de comunicación previstos. Además, se observa el papel mediador que ejerce la calidad de la relación en el efecto de la comunicación sobre dicha transferencia.

Así pues, el desarrollo de relaciones satisfactorias entre estos socios, y, por ende, el logro de una transferencia de conocimiento satisfactoria, viene determinado por la calidad de la relación que mantienen. De este modo, un clima de confianza, compromiso y relacionismo se asocia con una relación más exitosa, un mejor desempeño de sus tareas y, en general, una mayor satisfacción de los socios con la relación, que describe una satisfactoria transferencia de conocimiento entre el franquiciador y los franquiciados.

\section{Conclusiones}

La franquicia constituye una forma exitosa de expansión de un concepto de negocio. En este sistema de cooperación es clara la relación contractual existente entre las partes, si bien cobra una gran importancia el desarrollo de los vínculos relacionales entre franquiciador y franquiciados por su influencia en los resultados de tal cooperación a largo plazo. En este sentido, favorecer adecuados procesos de comunicación para lograr una transferencia de conocimiento satisfactoria entre los socios resulta fundamental. A su vez, en este proceso el desarrollo de una cultura basada en la confianza, el compromiso y el relacionismo, que define la calidad de la relación, ejerce un importante papel mediador. En esta investigación se observa el impacto de la comunicación entre el franquiciador y el franquiciado, como socios de un acuerdo de cooperación, en el logro de una satisfactoria transferencia de conocimiento, tal y como se planteó en el modelo propuesto.

En consecuencia, entre las principales implicaciones para la gestión directiva, cabe señalar la importancia de que el franquiciador preste una atención especial a la comunicación. Esto se puede canalizar a través del diseño y aplicación de un sistema de información que permita recoger, analizar y difundir la información necesaria a todos sus franquiciados para conseguir alcanzar sus objetivos, lo que requiere establecer un sistema sustentado en el intercambio mutuo y frecuente de la información necesaria y beneficiosa para la relación, así como favorecer una comunicación de calidad. Unos adecuados sistemas de información y comunicación favorecerán el acceso a los conocimientos, capacidades y otros recursos distintivos y complementarios de los socios de la franquicia, propiciando así la coordinación necesaria para que la organización pueda operar de forma eficiente y desempeñe con éxito su actividad.

Por su parte, dada la importancia de la calidad de la relación como elemento mediador entre la comunicación y la transferencia de conocimiento satisfactoria, se concluye que las empresas franquiciadoras deberían diseñar programas dirigidos a establecer, mantener y potenciar sus relaciones con los franquiciados, fomentando una cultura basada en la confianza, el compromiso y el relacionismo entre ambas partes. Para crear el cli- 
ma de confianza necesario se debe impulsar valores como la credibilidad, la honestidad y la sinceridad. En cuanto al compromiso, es preciso promover valores como la lealtad, la fidelidad y la colaboración. Finalmente, para alcanzar un alto grado de relacionismo, se ha de desarrollar un clima sustentado en la solidaridad y la mutualidad, de tal forma que tanto franquiciador como franquiciado consideren la relación como un "esfuerzo de cooperación" más que como un "conjunto de transacciones individuales".

En definitiva, en la medida en que se fomente en la franquicia una comunicación bidireccional, frecuente, adecuada, completa y oportuna, que genere un clima de confianza, compromiso y relacionismo, se alcanzará una satisfactoria transferencia de conocimiento. Esto sugiere como conclusión que una mayor honestidad, fiabilidad y compromiso de los socios en la relación, entre otros aspectos, favorecerá el desarrollo de relaciones satisfactorias de colaboración entre ellos y, consecuentemente, su disposición a volver a hacer negocios con las mismas empresas participantes, al no estar interesados en ofertas de otras empresas. Cabría cuestionarse si tales relaciones satisfactorias pueden asociarse con el logro de ventajas competitivas, objetivos estratégicos y resultados económico-financieros, en términos de beneficios y rentabilidad, lo que podría ser objeto de estudio en futuras investigaciones.

A pesar de todas estas aportaciones, es preciso reconocer ciertas limitaciones del estudio: (1) el uso del cuestionario como instrumento de recogida de la información, por lo que en el futuro se abordarían estudios de naturaleza cualitativa con el fin de profundizar en la problemática analizada; (2) la realización de la encuesta únicamente por una de las partes de la relación objeto de estudio, por lo que las investigaciones futuras recogerían el análisis y la evaluación de esta relación desde la perspectiva de los franquiciados, y (3) la medida utilizada para evaluar la transferencia de conocimiento satisfactoria, que podría complementarse con otros indicadores como el coste y el tiempo necesario para la transferencia o el impacto en los resultados organizativos que conlleva la transferencia de conocimiento, lo que sugiere el desarrollo de esta línea de investigación.

\section{Referencias Bibliográficas}

Albino, Vito; Garavelli, Achille Claudio y Schiuma, Giovanni (2001). A metric for measuring knowledge codification in organisation learning, Technovation, Vol. 21, pp. 413-422.

Anderson, Erin y Weitz, Barton (1992). The use of pledges to build and sustain commitment in distribution Channels, Journal of Marketing Research, Vol. 29 (febrero), pp. 18-34.

Baker, Thomas L.; Simpson, Penny M. y Siguaw, Judy A. (1999). The impact of suppliers' perceptions of reseller market orientation on key relationship constructs, Journal of the Academy of Marketing Science, Vol. 27, No. 1, pp. 50-57.

Bayona, Cristina; García-Marco, Teresa y Huerta, Emilio (2001). Firms' motivations for cooperative R\&D: an empirical analysis of Spanish firms, Research Policy, No. 30, pp. 12891307. 
Bello, Daniel C.; Chelariu, Cristian y Zhang, Li (2003). The antecedents and performance consequences of relationism in export distribution channels, Journal of Business Research, Vol. 56, pp. 1-16.

Benavides Espinosa, María del Mar (2011). El conocimiento como recursos estratégico para el aprendizaje interorganizativo, Revista Venezolana de Gerencia, Vol. 16, No. 56, pp. 564-577.

Bermúdez González, Guillermo José (2002). La franquicia: elementos, relaciones y estrategias. Madrid, España, ESIC.

Bordonaba Juste, María Victoria y Polo Redondo, Yolanda (2004). Gestión estratégica de la relación de franquicia, Universia Business Review, No. 1, pp. 50-63.

Bordonaba Juste, María Victoria; Lucía Palacios, Laura y Polo Redondo, Yolanda (2009). Evolución del sistema de franquicia en España: un estudio en los sectores de moda y hostelería, Distribución y Consumo, No. 105, pp. 6476.

Boyle, Brett A. y Dwyer, F. Robert (1995). Power, bureaucracy, influence, and performance: their relationships in industrial distribution channels, Journal of Business Research, Vol. 32, pp. 189-200.

Boyle, Brett A.; Dwyer, F. Robert; Robicheaux, Robert A. y Simpson, James T. (1992). Influence strategies in marketing channels: Measures and use in different relationship structures, Journal of Marketing Research, Vol. 29 (noviembre), pp. 462-473.

Brown, James R.; Lusch, Robert F. y Nicholson, Carolyn Y. (1995). Power and relationship commitment: their impact on marketing channel member performance, Journal of Retailing, Vol. 71, No. 4, pp. 363-392.
Cabrera, Ángel y Cabrera, Elizabeth F. (2002). Knowledge-sharing dilemmas, Organization Studies, Vol. 23, No. 5, pp. 687-710.

Cannon, Joseph P.; Achrol, Ravi S. y Gundlach, Gregory T. (2000). Contracts, norms, and plural form governance, Journal of the Academy of Marketing Science, Vol. 28, No. 2, pp. 180-194.

Cannon, Joseph P. y Perreault, William D. (1999). Buyer-seller relationships in business markets, Journal of Marketing Research, Vol. 36 (noviembre), pp. 439-460.

Cassiman, Bruno (1999). Cooperación en investigación y desarrollo. Evidencia para la industria manufacturera española, Papeles de Economía Española, No. 81, pp. 143-154.

Cepeda Pérez, Juan Manuel (2003). El canal de distribución: disección de las relaciones comerciales. Oviedo, España, Septem Ediciones.

Combs, James G. y Castrogiovanni, Gary J. (1994). Franchisor strategy: A proposed model and empirical test of franchise versus company ownership, Journal of Small Business Management, Vol. 32, No. 2, pp. 37-48.

Cummings, Jeffrey L. y Teng, Bing-Sheng (2003). Transferring R\&D knowledge: the key factors affecting knowledge transfer success, Journal of Engineering and Technology Management, Vol. 20, pp. 39-68.

Daft, Richard L. y Lengel, Robert H. (1986). Organizational information requirements, media richness and structural design, Management Science, Vol. 32, No. 5, pp. 554-571.

Das, T. K. y Teng, Bing-Sheng (1998). Between trust and control: developing confidence in partner cooperation in alliances, Academy of Management Review, Vol. 23, No. 3, pp. 491-512. 
Dawson, Ross (2000). Knowledge capabilities as the focus of organizational development and strategy, Journal of Knowledge Management, Vol. 4, No. 4 , pp. 320-327.

De Wulf, Kristof; Odekerken-Schröder, Gaby y Lacobucci, Dawn (2001). Investments in consumer relationships: a crosscountry and cross-industry exploration, Journal of Marketing, Vol. 65 (October), pp. 33-50.

Díez, Enrique Carlos y Galán, José Luis (1998). Práctica de la franquicia. Madrid, España, McGraw-Hill.

Doney, Patricia M. y Cannon, Joseph P. (1997). An examination of the nature of trust in buyer-seller relationships, Journal of Marketing, Vol. 61, pp. 35-51.

Feng, Changli y Zhang, Tong (2007). Trust and knowledge transfer between business partners: the mediating effects of three factors, Proceedings of the IEEE International Conference on Automation and Logistics, Jinan, China.

Ganesan, Shankar (1994). Determinants of long-term orientation in buyer-seller relationships, Journal of Marketing, Vol. 58, pp. 1-19.

Geyskens, Inge; Steenkamp, Jan-Benedict E. M.; Scheer, Lisa K. y Kumar, Nimalya (1996). The effects of trust and interdependence on relationship commitment: a trans-atlantic study, International Journal of Research in Marketing, Vol. 13, pp. 303-317.

Gilliland, David I. y Bello, Daniel C. (2002). Two sides to attitudinal commitment: the effect of calculative and loyalty commitment on enforcement mechanisms in distribution channels, Journal of the Academy of Marketing Science, Vol. 30, No. 1, pp. 24-43.

Goh, Swee C. (2002). Managing effective knowledge transfer: an integrative framework and some practice implica- tions, Journal of Knowledge Management, Vol. 6, No. 1, pp. 23-30.

Gulati, Ranjay (1995). Does familiarity breed trust? The implications of repeated ties for contractual choice in alliances, Academy of Management Journal, Vol. 38, No. 1, pp. 85-112.

Hair, Joseph; Anderson, Rolph; Tatham, Ronald y Black, William (1999). Análisis multivariante. $5^{\text {a }}$ edición, Madrid, España, Prentice Hall.

Hamel, Gary; Doz, Yves L. y Prahalad, C. K. (1989). Collaborate with your competitors and win, Harvard Business Review, Vol. 67, pp. 133-139.

Hausman, Angela (2001). Variations in relationship strength and its impact on performance and satisfaction in business relationships, Journal of Business \& Industrial Marketing, Vol. 16, No. 7, pp. 600-616.

Heide, Jan B. y John, George (1992). Do norms matter in marketing relationship?, Journal of Marketing, Vol. 56, pp. 32-44.

Jap, Sandy D. y Ganesan, Shankar (2000). Control mechanisms and the relationship life cycle: implications for safeguarding specific investments and developing commitment, Journal of Marketing Research, Vol. 37, pp. 227-245.

Jap, Sandy D.; Manolis, Chris y Weitz, Barton A. (1999). Relationship quality and buyer-seller interactions in channels of distribution, Journal of Business Research, Vol. 46, pp. 303-313.

Kogut, Bruce y Singh, Harbir (1988). The effect of national culture on the choice of entry mode, Journal of International Business Studies, Vol. 19, pp. 411432.

Kumar, Nirmalya; Scheer, Lisa K. y Steenkamp, Jan-Benedict E. M. (1995). The effects of perceived interdependence on dealer attitudes, Journal of Mar- 
keting Research, Vol. 32, pp. 348356.

Leonard-Barton, Dorothy y Sinha, Deepak K. (1993). Developer-user interaction and user satisfaction in internal technology transfer, Strategic Management Journal, Vol. 36, pp. 11251139.

Lusch, Robert F. y Brown, James R. (1996). Interdependency, contracting, and relational behavior in marketing channels, Journal of Marketing, Vol. 60, pp. 19-38.

Mayer, Roger C., Davis, James H. y Schoorman, F. David (1995). An integrative model of organizational trust, Academy of Management Review, Vol. 20, No. 3, pp. 709-734.

Mohr, Jakki J. y Nevin, John R. (1990). Communication strategies in marketing channels: a theoretical perspective, Journal of Marketing, Vol. 54, pp. 36-51.

Mohr, Jakki J. y Sohi, Ravipreet S. (1995). Communication flows in distribution channels: impact on assessments of communication quality and satisfaction, Journal of Retailing, Vol. 71, No. 4, pp. 393-416.

Mohr, Jakki J. y Spekman, Robert (1994). Characteristics of partnership success: partnership attributes, communication behavior, and conflict resolution techniques, Strategic Management Journal, Vol. 15, pp. 135-152.

Mohr, Jakki J.; Fisher, Robert J. y Nevin, John R. (1996). Collaborative communication in interfirm relationships: moderating effects of integration and control, Journal of Marketing, Vol. 60, pp. 103-115.

Montoro-Sánchez, Ángeles; Mora-Valentín, Eva María y Guerras-Martín, Luis Ángel (2010). Trust matters in cooperative agreements but does the nature of the nature of the partner also matter? Academia, Revista Latinoamericana de Administración, Vol. 45, pp. 96-115.

Morgan, Robert M. y Hunt, Shelby D. (1994). The commitment-trust theory of relationship marketing, Journal of Marketing, Vol. 58, pp. 20-38.

Mothe, Caroline y Quélin, Bertrand (2000). Creating competencies through collaboration: the case of EUREKA R\&D Consortia, European Management Journal, Vol. 18, No. 6, pp. 590-605.

Norton, Seth W. (1988). Franchising, brand name capital, and the entrepreneurial capacity problem, Strategic Management Journal, Vol. 9, No. S1, pp. 105-114.

Oxenfeldt, Alfred R. y Kelly, Anthony O. (1969). Will successful franchise systems ultimately become wholly-owned chains?, Journal of Retailing, Vol. 44, pp. 69-83.

Paswan, Audesh K. y Young, Joyce A. (1999). An exploratory examination of the relationship between channel support mechanisms and relational norms in an international context, Journal of Business \& Industrial Marketing, Vol. 14, No. 5/6, pp. 445-455.

Pedersen, Torben; Petersen, Bent y Sharma, Deo (2003). Knowledge transfer performance of multinational companies, Management International Review, Vol. 43, No. 3, pp. 69-90.

Peña Legazkue, Iñaki y Aranguren Querejeta, María José (2002). Transferencia de conocimiento mediante acuerdos de cooperación, Economía Industrial, No. 346, pp. 67-80.

Reddy, N. Mohan y Zhao, Liming (1990). International technology transfer, Research Policy, Vol. 19, pp. 285-307.

Rodríguez Benavides, María Concepción; Minguela Rata, Beatriz y López Sánchez, José Ignacio (2008). Transfe- 
rencia de conocimiento en la creación y el funcionamiento de los sistemas de franquicia: un estudio empírico, Economía Industrial, No. 368, pp. 227-237.

Rodríguez Orejuela, Augusto (2007). Transferencia de conocimiento en relaciones inter-organizacionales: su efecto sobre el desempeño de la firma receptora, Estudios Gerenciales, Vol. 23, No. 103, pp. 13-37.

Sakakibara, Mariko (1997). Heterogeneity of firm capabilities and cooperative research and development: and empirical examination of motives, Strategic Management Journal, Vol. 18 (summer special issue), pp. 143-164.

Santamaría Sánchez, Lluis y Rialp Criado, Josep (2007). La elección del socio en las cooperaciones tecnológicas: un análisis empírico, Cuadernos de Economía y Dirección de Empresas, Vol. 31, pp. 67-97.

Sarkar, MB; Echambadi, Raj; Cavusgil, S. Tamer y Aulakh, Preet S. (2001). The influence of complementarity compatibility and relationship capital on alliance performance, Journal of the Academy of Marketing Science, Vol. 29, No. 4, pp. 358-373.

Selnes, Fred (1998). Antecedents and consequences of trust and satisfaction in buyer-seller relationships, European Journal of Marketing, Vol. 32, No. 3/4, pp. 305-322.

Siguaw, Judy A.; Simpson, Penny M. y Baker, Thomas L. (1998). Effects of supplier market orientation on distributor market orientation and the channel relationship: the distributor perspective, Journal of Marketing, Vol. 62, pp. 99-111.

Simpson, James T. y Mayo, Donna T. (1997). Relationship management: a call for fewer influence attempts? Journal of Business Research, Vol. 39, pp. 209-218.

Szulanski, Gabriel (1995). Appropriating rents from existing knowledge: intra-firm transfer of best practice. UMI Dissertation Services.

Teece, David J. (1977). Technology transfer by multinational corporations: the resource cost of transferring technological know-how, Economic Journal, Vol. 87, pp. 242-261.

Verhoef, Peter C.; Franses, Philip Hans y Hoekstra, Janny C. (2002). The effect of relational constructs on customer referrals and number of services purchased from a multiservice provider: does age of relationship matter?, Journal of the Academy of Marketing Science, Vol. 30, No. 3, pp. 202216.

Williams, Frederick y Gibson, David V. (1990). Technology transfer: a communication perspective. Estados Unidos, Sage Publications.

Zander, Udo y Kogut, Bruce (1995). Knowledge and the speed of transfer and imitation of organizational capabilities: an empirical test, Organization Science, Vol. 6, No. 1, pp. 76-92. 